\title{
Mid Transverse Colon Cancer and the Related Risk Factors: A Case Report
}

Amer Hayat Khan ${ }^{1 *}$, Andee Dzulkarnaen Zakaria², Syed Hassan², Siti Rahmah Hashim Isa Merican², Nurashikin Binti Mazlan ${ }^{1}$ and Mohammed Abdul Hameed ${ }^{3}$

${ }^{1}$ Department of Clinical Pharmacy, School of Pharmaceutical Sciences Universiti Sains Malaysia, 11800 Penang, Malaysia ${ }^{2}$ Department of Surgery, School of Medical Sciences, Universiti Sains Malaysia, Health Campus 16150 Kelantan, Malaysia

\begin{abstract}
Background: Colorectal cancer is the third most common cancer among both men and women in the United States. A colorectal cancer begins when normal cells in the lining of the colon or rectum change and grows uncontrollably, forming a mass called a tumor.

Case presentation: A 54-year-old woman complained of epigastric pain for the last four months and the pain increased in severity in the last few days. Computed tomography (CT) scan showed mid transverse colon tumor with the absence of pericolic fat streakiness, lymphodenopathy and liver metastases. The patient has diabetes mellitus (DM) type 2 and hypertension. She has been on insulin therapy for years (subcutaneous (S/C) Actrapid $30 \mathrm{u}$ TDS and S/C Insulatard $8 \mathrm{u}$ ON). Patient is obese with BMI of $36.5 \mathrm{~kg} / \mathrm{m} 2$. The colonoscopy and pathological examination showed polypoidal tumour (Transverse) with length of $5.5 \mathrm{~cm}$, the lumen of the colon is almost completely obstructed by the tumour. The patient underwent right hemicolectomy and her condition improved afterwards.
\end{abstract}

Conclusion: Diabetes Mellitus and aggressive insulin therapy may be the underlying factors that causing emergence of colon cancer in current patient, even though there is no family history for cancer.

Keywords: Colorectal cancer; Diabetes mellitus; Drug therapy; Hypertension; Surgery

\section{Introduction}

Colorectal cancer is associated with over 1.2 million new cancer cases and 608,700 deaths estimated to have occurred worldwide in 2008 [1]. In Malaysia, colorectal cancer is the third commonest cause of cancer deaths. Data from the Ministry of Health of Malaysia confirms an increase in colorectal cancer admission rates and considered one of 5 most common cancer in Malaysia [2].

One of the common types of tumor in the colon is known as polyps. Polyps are abnormal growths that protrude from the inner wall of the colon or rectum [3]. They are relatively common in people over age 50. Most polyps are noncancerous, but majority of colorectal cancer is believed to develop in polyps known as adenomas [3].

The probability of polyps to be detected as colon cancer is directly related to size the polyps. Two percent (2\%) of colon cancer develops in the polyps size $0.6-1.5 \mathrm{~cm}, 19 \%$ between $1.6-2.5 \mathrm{~cm}, 43 \%$ is between $2.6-3.5 \mathrm{~cm}$ and $76 \%$ in more than $>3.5 \mathrm{~cm}$ polyps [3].

The studies have shown that certain factors are linked to an increased chance of developing colon cancer including age (over 50 years old), existence of polyps in the colon/rectum, personal/family history of colorectal cancer, ulcerative colitis, unhealthy diet, sedentary lifestyle and smoking. 3 Besides, there are some factors linked to colon cancer, one of which are diseases such as diabetes, as well as certain course of treatment such as aggressive long duration insulin treatment [4].

\section{Case Summary}

A 54-year-old Malay woman presented with epigastric pain for 4 months duration, the severity increased since few days before admission. It was sudden in onset, colicky in nature, radiated to the middle of the chest and associated with vomiting which contained food particle. The pain was not associated with meal. There was no history of jaundice; chest pain, palpitation or breathlessness, and even patient denied any family history of colorectal neoplasia. There was change in bowel habit in which patient was having constipation and diarrhea frequently. This patient had underlying hypertension and insulin dependent diabetes mellitus.

Patient underwent ultrasonography and the result is normal. Computed Tomography (CT) scan of the colon showed a circumferiantial narrowing of the transverse colon at the mid part of it with the length of $5.5 \mathrm{~cm}$ (Figure 1). There was presence of dilated bowel proximal to the narrowing with multiple air fluid level. Furthermore, there is a noted small bowel dilatation but however the cause was unknown. There was no lymphadenopathy or evidence of distant metastasis. She underwent right hemicolectomy and was discharged home after several days.

\section{Discussion}

The pathological examination showed polypoidal tumour of the colon with length of $5.5 \mathrm{~cm}$ and the lumen of the colon is almost completely obstructed by the tumour. Based on the length of the tumour, there is a more than $76 \%$ risk that the tumour found is cancerous in nature. Genetics, experimental, and epidemiological data suggest that colon cancer develops from complex interactions between inherited susceptibility and environmental factors [2]. The commonly heard risk factors for colon cancers include age (over 50 years old),

*Corresponding author: Amer Hayat Khan, Department of Clinical Pharmacy, School of Pharmaceutical Sciences, Universiti Sains Malaysia, 11800 Penang, Malaysia, Tel: 006-014-9227580; Fax: 00604657 0017; E-mail: amerhayat@ymail.com

Received January 15, 2013; Accepted July 13, 2013; Published July 17, 2013

Citation: Khan $A H$, Zakaria AD, Hassan S, Isa Merican SRH, Mazlan NB, et al. (2013) Mid Transverse Colon Cancer and the Related Risk Factors: A Case Report. Trop Med Surg 1: 130. doi:10.4172/2329-9088.1000130

Copyright: ( $2013 \mathrm{Khan} \mathrm{AH}$, et al. This is an open-access article distributed unde the terms of the Creative Commons Attribution License, which permits unrestricted use, distribution, and reproduction in any medium, provided the original author and source are credited. 


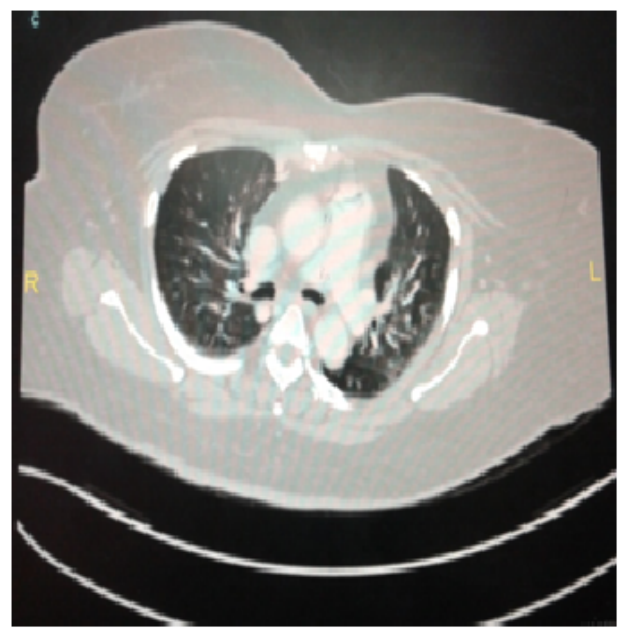

Figure 1: Computed Tomography (CT) scan of the colon showed a circumferiantial narrowing of the transverse colon at the mid part of it with the length of $5.5 \mathrm{~cm}$.

existence of adenomatous polyps in the colon, personal/family history of colorectal cancer, ulcerative colitis, diet (high dietary consumption of processed meat and low consumption of fibres), sedentary lifestyle and smoking. Furthermore, there are some other silent contributing factors for developing colon cancer like comorbid conditions and drug therapy [3]. Literature revealed the association persisted even after adjustment of other colorectal cancer risk factors like body mass index (BMI), physical activity, screening patterns, and dietary factors [5].

Patient has been suffering from Diabetes Mellitus Type 2 for many years. Some studies had suggested that there is strong evidence to indicate that Diabetes Mellitus is causal agents for colon cancer development [6]. Nevertheless, a large number of epidemiological studies have demonstrated that the risk for colon cancer increased in patients with type $2 \mathrm{DM}$. A recent meta-analysis showed that patients with type $2 \mathrm{DM}$ have a $30 \%$ increased risk for colon cancer versus the general population and that this risk is doubled in diabetic patients treated with insulin [7-10].

Other than as a risk factor for colon cancer, a study has proven that patients with diabetes mellitus and high-risk stage II and stage III colon cancer experienced a significantly higher rate of overall mortality and cancer recurrence, even after adjustment for other predictors of colon cancer outcome [8]. Patients without DM is found to have better outcome compared to those with underlying DM.

Patient has been on insulin therapy [Actrapid, (short acting insulin) and Insulatard (long acting insulin)], for years. A study has proved that chronic insulin therapy significantly increases the risk of colorectal cancer among type 2 diabetes mellitus patients $[9,10]$. Insulin is an important growth factor of colonic epithelial cells and is a mitogen of tumor cell growth in vitro. Epidemiologic evidence supporting the insulin/colon-cancer hypothesis is largely indirect and based on the similarity of factors which produce elevated insulin levels with those related to colon cancer risk.

Not all anti-diabetic therapy will increase the risk for colon cancer. There is a study done to evaluate the association between metformin and colon cancer risk among patients with type 2 diabetes. The results indicate that metformin therapy was associated with an estimated reduction of $37 \%$ in the risk of colon cancer among patients with type
2 diabetes. In vitro studies have shown that metformin inhibits the proliferation of colon cancer cells [11].

Current patient was also on Lovastatin. The use of statins has been associated with a small reduction in the risk for colon cancer in diabetic patients [1].

\section{Conclusion}

Colon cancer is claimed to be caused by the effects of industrialization, smoking. Diabetes mellitus and insulin therapy might be the important factors that may have contribute to the development of colon cancer. Comorbid or multiple comorbid condition leads to many drug regimens which need proper attention to avoid future complications like Lovastatin in current prescription for such patient.

\section{References}

1. Jemal A, Bray F, Center MM, Ferlay J, Ward E, et al. (2011) Global cancer statistics. CA Cancer J Clin 61: 69-90.

2. Malaysian Cancer Statistics-Data and Figure Peninsular Malaysia 2006 National Cancer Registry, Ministry of Health Malaysia. Kuala Lumpur: 2006; 8-9.

3. National Cancer Institute of United States. Colorectal Cancer Screening. 30th December 2011. Available Online; URL: http://www.cancer.gov/cancertopics/ factsheet/detection/colorectal-screening/print (accessed 6 July 2012)

4. Giouleme O, Diamantidis MD, Katsaros MG (2011) Is diabetes a causal agent for colorectal cancer? Pathophysiological and molecular mechanisms. World J Gastroenterol 17: 444-448.

5. Meyerhardt JA, Catalano PJ, Haller DG, Mayer RJ, Macdonald JS, et al. (2003) Impact of diabetes mellitus on outcomes in patients with colon cancer. $\mathrm{J}$ Clin Oncol 21: 433-440.

6. Zuber M. and Harder F. Benign tumors of the colon and rectum. W. Zuckschwerdt Verlag GmbH. Switzerland: 2001.

7. Meyerhardt JA, Catalano PJ, Haller DG, Mayer RJ, Macdonald JS, et al. (2003) Impact of diabetes mellitus on outcomes in patients with colon cancer. $\mathrm{J}$ Clin Oncol 21: 433-440.

8. Giovannucci E (1995) Insulin and colon cancer. Cancer Causes Control 6: 164 179

9. Yang YX, Hennessy S, Lewis JD (2004) Insulin therapy and colorectal cancer risk among type 2 diabetes mellitus patients. Gastroenterology 127: 1044-1050.

10. Jeon JY, Jeong DH, Park MG, Lee JW, Chu SH, et al. (2013) Impact of diabetes on oncologic outcome of colorectal cancer patients: colon vs. rectal cancer PLoS One 8: e55196.

11. Zhang ZJ, Zheng ZJ, Kan H, Song Y, Cui W, et al. (2011) Reduced risk of colorectal cancer with metformin therapy in patients with type 2 diabetes: a meta-analysis. Diabetes Care 34: 2323-2328. 